\title{
Changes in the mitochondrial network during ectromelia virus infection of permissive L929 cells*
}

\author{
Karolina P. Gregorczyk, Lidia Szulc-Dąbrowska, Zbigniew Wyżewski, Justyna Struzik and \\ Marek Niemiałtowski ${ }^{\bowtie}$ \\ Division of Immunology, Department of Preclinical Sciences, Faculty of Veterinary Medicine, Warsaw University of Life Sciences - SGGW, War- \\ szawa, Poland
}

\begin{abstract}
Mitochondria are extremely important organelles in the life of a cell. Recent studies indicate that mitochondria also play a fundamental role in the cellular innate immune mechanisms against viral infections. Moreover, mitochondria are able to alter their shape continuously through fusion and fission. These tightly regulated processes are activated or inhibited under physiological or pathological (e.g. viral infection) conditions to help restore homeostasis. However, many types of viruses, such as orthopoxviruses, have developed various strategies to evade the mitochondrial-mediated antiviral innate immune responses. Moreover, orthopoxviruses exploit the mitochondria for their survival. Such viral activity has been reported during vaccinia virus (VACV) infection. Our study shows that the Moscow strain of ectromelia virus (ECTV-MOS), an orthopoxvirus, alters the mitochondrial network in permissive L929 cells. Upon infection, the branching structure of the mitochondrial network collapses and becomes disorganized followed by destruction of mitochondrial tubules during the late stage of infection. Small, discrete mitochondria co-localize with progeny virions, close to the cell membrane. Furthermore, clustering of mitochondria is observed around viral factories, particularly between the nucleus and viroplasm. Our findings suggest that ECTV-MOS modulates mitochondrial cellular distribution during later stages of the replication cycle, probably enabling viral replication and/or assembly as well as transport of progeny virions inside the cell. However, this requires further investigation.
\end{abstract}

Key words: mitochondrial network, ECTV-MOS, apoptosis

Received: 07 November, 2013; revised: 20 February, 2014; accepted: 24 February, 2014; available on-line: 22 March, 2014

\section{INTRODUCTION}

Ectromelia virus (ECTV) is an enveloped DNA virus that belongs to the Orthopoxvirus genus of the Poxviridae family. It is closely related to variola virus (VARV), a causative agent of smallpox, an infectious disease with the highest number of fatalities ever (McFadden, 2005). Vaccinia virus (VACV), also an orthopoxvirus, has been used successfully as a vaccine to eradicate smallpox (Fenner, 2000). ECTV causes mousepox in various breeds of mice. Because symptoms of mousepox are reminiscent of smallpox in humans, the disease in mice is occasionally referred to as "smallpox of mice". However, ECTV does not cause disease in humans. For that reasons,
ECTV has repeatedly been used as a model to study pathogenesis of orthopoxviruses with the aim of elucidating mechanisms of host defense against these viruses.

Mitochondria are key organelles in eukaryotic cells. They are involved in a range of cellular metabolic processes, such as cellular energy supply, cellular differentiation, aging, calcium buffering to red-ox homeostasis, as well as cell death. In addition to their role in elimination of infected cells through apoptosis, mitochondria contribute to interferon (IFN)-signaling, leading to production of type I IFNs, thus indicating their function in the primary host defense against viral infections (Tait \& Green, 2010). Numerous orthopoxviral genes modulate host immunity including mitochondrial-mediated antiviral response (Melo-Silva, 2011). For instance, they can cordon off mitochondria to prevent the release of mediators of apoptosis and/or activation of IFN production. Furthermore, poxviruses can exploit mitochondria for their survival through viral protein-mediated increase in concentration of mitochondria near the viral factories for the purpose of providing energy during viral replication and/or morphogenesis. An example of such evasive strategy is exhibited by VACV through extensive modification of the intracellular distribution of mitochondria during infection (Schepis et al., 2006).

Mitochondria are highly dynamic organelles (BereiterHahn \& Voth, 1994). In many cell types, mitochondria have a tubular morphology; they form a branched, interconnecting network and undergo morphological and functional changes due to their frequent fusion and fission in response to local environmental conditions. The fusion/fission machinery is represented by the mitochondria shaping proteins, such as mitofusin 1 (Mfn1), mitofusin 2 (Mfn2), optic atrophy 1 (Opa1), regulators of fusion, and dynamin related protein 1 (Drp1), fission protein 1 (Fis1), mitochondrial fission factor (Mff), and mitochondrial elongation factor 1 (MIEF1), which modulate fission (Bereiter-Hahn \& Voth, 1994). During the fusion/fission processes mitochondria can shift between large, branched networks and small, circular or fragmented units. Cells undergoing mitochondrial fission have mitochondria shorter in length when compared to cells that are undergoing mitochondrial fusion. The balance between fusion and fragmentation is important to main-

e-mail: marek_niemialtowski@sggw.pl

*Presented at the 3rd Workshop on Microbiology in Health and Environmental Protection "MIKROBIOT 2013", Łódź, Poland

Abbreviations: ASFV, African swine fever virus; ECTV, ectromelia virus; ECTV-MOS, Moscow strain of ECTV; IFN, interferon; MTOC, microtubule organizing center; ROS, reactive oxygen species; RER, rough endoplasmic reticulum; VACV, vaccinia virus 
tain normal mitochondrial size, shape and distribution, and to supply energy to high-demand sites. Significantly, the cytoskeleton interacts with mitochondria and is responsible for mitochondrial transport throughout the cytoplasm (Boldogh \& Pon, 2006; Guzun et al., 2011).

Normally, mitochondria are tubular in shape but may attain various forms under different pathological conditions (stress) (Wasilewski \& Scorrano, 2009; Arnoult, 2007). There are three main types of mammalian cell deaths, which can be characterized by different mitochondrial morphology. Fragmented, round tubes or blob-shaped mitochondria are observed during apoptosis, whereas swollen mitochondria are characteristic for necrosis (Ahmad et al., 2013) and in autophagy mitochondria become elongated and are spared from degradation (Gomes, 2011). Overall, mitochondria can form circular tubes with a clear center sometimes referred to as donut-shaped mitochondria (Benard et al., 2007; Liu \& Hajnóczky, 2011), which according to a recent study may indicate their role in the mitochondrial adaptation to hypoxia-reoxygenation-induced cellular stress (Liu \& Hajnóczky, 2011).

In the present study we evaluated the mitochondrial network organization in L929 cells infected with Moscow strain of ECTV (ECTV-MOS). Our results show that upon infection, the branching structure of mitochondrial network collapses and becomes disorganized. Significantly, mitochondrial tubules are destroyed during the late stage of infection. Moreover small, discrete mitochondria co-localize with viral progeny close to the cell membrane. Furthermore, clustering of mitochondria is observed around viral factories, particularly between the nucleus and viroplasm. Results are discussed in the context of poxvirus immune evasion mechanisms.

\section{MATERIALS AND METHODS}

Virus. ECTV-MOS was obtained from the American Type Culture Collection (ATCC 1374, Manassas, VA, USA), propagated and later titrated by plaque assay $(\mathrm{PFU} / \mathrm{ml}$ ) in Vero cell culture (kidney epithelial cells extracted from African green monkey; ATCC CCL-81) in DMEM medium containing L-glutamine (Gibco BRL, Grand Island, NY, USA), supplemented with 1\% fetal bovine serum (FBS; Sigma-Aldrich, St. Louis, MO, USA) and $1 \%$ antibiotic/antimycotic $(100 \mathrm{U} / \mathrm{ml}$ penicillin, 100 $\mu \mathrm{g} / \mathrm{ml}$ streptomycin, $250 \mathrm{ng} / \mathrm{ml}$ amfotericin-B) (SigmaAldrich) at $37^{\circ} \mathrm{C}$ in a humidified atmosphere with $5 \%$ $\mathrm{CO}_{2}$ in the air. The virus was purified and stored at $-70^{\circ} \mathrm{C}$

Cell culture. L929 cells (mouse fibroblastic cell line, haplotype $\mathrm{H}-2 \mathrm{k}$; ATCC CCL-1), which are permissive to ECTV infection, were used in this research. The L929 cells were cultivated in DMEM high glucose medium containing $4.0 \mathrm{mM}$ L-glutamine (Gibco BRL) and supplemented with 5\% FBS and 1\% antibiotic/antimycotic. Twenty-four hour cell culture was infected with ECTVMOS at the multiplicity of infection (M.O.I.) of 1 in culture medium supplemented with 1\% FBS and 1\% antibiotic/antimycotic. Uninfected (culture medium without ECTV-MOS) L929 cells were used as a negative control. At 4, 8, 18, and $24 \mathrm{~h}$ post-infection (h.p.i.), the cells were harvested for further analysis. Each experiment was performed in triplicate.

Mitochondria labeling with MitoTracker Red. L929 cells seeded on glass coverslips in a 24-well plate were infected with ECTV-MOS. At 4, 8, 18 or 24 h.p.i. cells were incubated for $30 \mathrm{~min}$ at $37^{\circ} \mathrm{C}$ with $100 \mathrm{nM}$
MitoTracker Red CMXRos (Molecular Probes, Inc., Eugene, OR, USA), washed three times with culture medium and fixed with 4\% paraformaldehyde (PFA, SigmaAldrich) or methanol (Sigma-Aldrich).

Immunofluorescent staining. Intracellular staining of viral proteins was performed as previously described (Boratynska et al., 2010). Briefly, fixed L929 cells were permeabilized with $0.5 \%$ Triton X-100 (Sigma-Aldrich) and blocked with 3\% bovine serum albumin (BSA, Sigma-Aldrich) to prevent nonspecific binding. The cells were subsequently incubated with rabbit antibodies directed against ECTV-MOS conjugated with FITC or unconjugated mouse anti- $\alpha$-tubulin monoclonal antibodies (Sigma-Aldrich). Next, secondary anti-mouse antibodies conjugated with FITC (Jackson ImmunoResearch) were used. The nuclei were stained with Hoechst 33342 (Sigma-Aldrich) and cells were mounted in ProLong Gold antifade reagent (Invitrogen, Carlsbad, CA, USA).

Fluorescence microscopy. Images were recorded using Olympus BX60 fluorescence microscope (Olympus, Tokyo, Japan) equipped with Color View III cooled $\mathrm{CCD}$ camera and processed using the Cell^ $\mathrm{F}$ (Olympus), CellSens Dimension (Olympus) and ImageJ (NIH Image, Bethesda, MD, USA) software.

\section{RESULTS AND DISCUSSION}

\section{Mitochondria display close association with ECTV-MOS factories in L929 cells}

Poxviruses are unique among most DNA viruses in that their replication occurs in the cytoplasm of the infected cells. The characteristic sites of replication and assembly are large cytoplasmic perinuclear areas termed viral factories, where viral and cell components move in and out depending on the stage of the viral replication. Studies on VACV have shown that the striking feature of these areas is their close association with the nucleus and mitochondria (Tolonen et al., 2001; Risco et al., 2002; Schepis et al., 2006).

Mitochondrial network in uninfected control L929 fibroblasts was characterized by extended wavy tubules distributed differently depending on a subcellular region of the cell (Fig. 1a). Near the nucleus (perinuclear region) mitochondria were arranged in a concentric manner, whereas radial distribution was observed closer to the cell membrane (periplasmic region). Moreover, the control cells clearly showed a microtubule organizing center (MTOC), from which mitochondrial tubules appear to radiate (Fig. 1b).

At the early stage of ECTV-MOS infection ( 4 h.p.i.) only a few cells had visible viral factories compared to vast majority of infected cells without viral factories and no significant alterations to the mitochondrial network structure (Fig. 2a). However, inconsiderable differences were noted in mitochondrial distribution compared to uninfected control cells (Figs. 1, 2). The noticeable accumulation of mitochondria around the nucleus at the presence of virions was observed (Fig. 2a). Interestingly, at 8 h.p.i we noticed formation of viral factories in the perinuclear region and the characteristic mitochondrial clustering around these areas of viral replication (Fig. 2b). Later, progressive changes occurred in mitochondrial distribution. Mitochondria began to accumulate between nucleus and viral factory/factories and disconnections between mitochondria within the cytoplasm occurred (Fig. 3a, 3d). At 18 and 24 h.p.i., viral factories lost their regular shape and took dispersed or expanded 
forms thus intensifying mitochondrial localization around viral replication/assembly sites (Fig. 3a, 3d).

Interestingly, MTOC gradually disappeared during ECTV-MOS infection (Figs. 1c, 2b, 3a, 3d) as opposed to MTOC in uninfected control cells which was intact and distinctly marked (Fig. 1b). We initially observed these events in BALB/3T3 and Vero cells during ECTV-MOS infection (data not shown). Additionally, viral particles and viral factories accumulate near MTOC in infected cells. ECTV-MOS virions co-localize with tubulin in BALB/3T3 and Vero cells (data not shown). Another study indicated that both mitochondria and virions localize to MTOC during African swine fever virus (ASFV) infection (Heath et al., 2001). Our observations confirm that mitochondria localize to MTOC in L929 cells during ECTV-MOS infection. Altogether, these findings suggest the participation of microtubules in the transport of viral particles as well as mitochondria during ECTV-MOS infection.

During VACV infection viral factories are enclosed by elements of the rough endoplasmic reticulum (RER) (Tolonen et al., 2001). Since close association between vi- ral factories and mitochondria was also indicated, it can be assumed that mitochondria interact with RER. It is well known that these structures collaborate during viral infection, because they are involved in the production of proinflammatory cytokines and type I IFNs (Ishikawa \& Barber, 2008). These findings may suggest that mitochondrial recruitment to viral factories is related to the early antiviral responses during VACV infection. It is highly possible that mitochondria engage in a similar mechanism in ECTV-MOS-infected L929 cells. However, VACV encodes proteins which inhibit mitochondria-mediated antiviral response (Deng et al., 2008; Chen et al., 2008). Only elongated mitochondria are able to transduce signals for production of type I IFNs and cytokines. Mitochondrial fragmentation leads to the loss of connection with endoplasmic reticulum and inhibits antiviral response. Fragmentation of mitochondrial network during VACV (Schepis et al., 2006) and ECTV-MOS infection indicates that these viruses may inhibit production of antiviral factors.

Our findings suggest that infection of L929 cells with ECTV-MOS rearranges mitochondrial distribu-

(a)
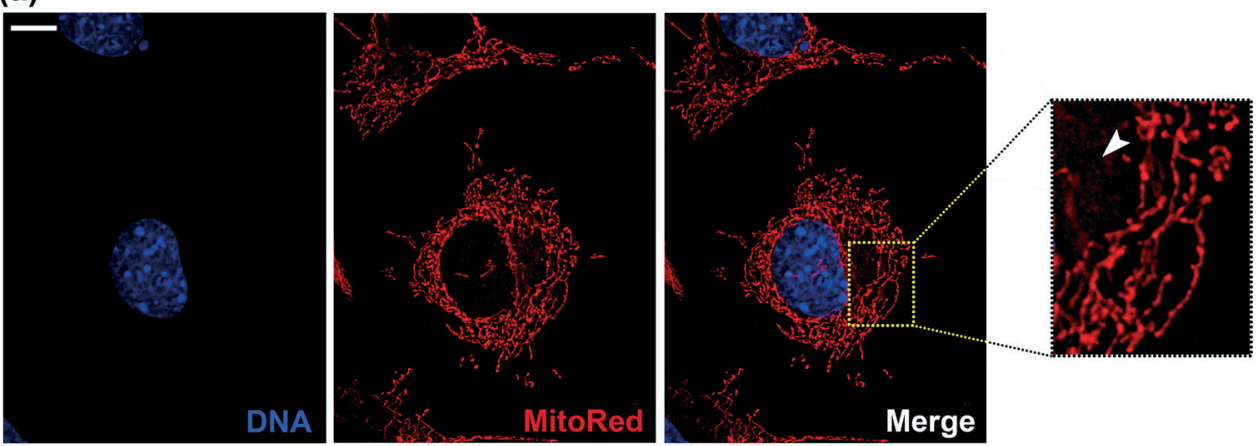

(b)
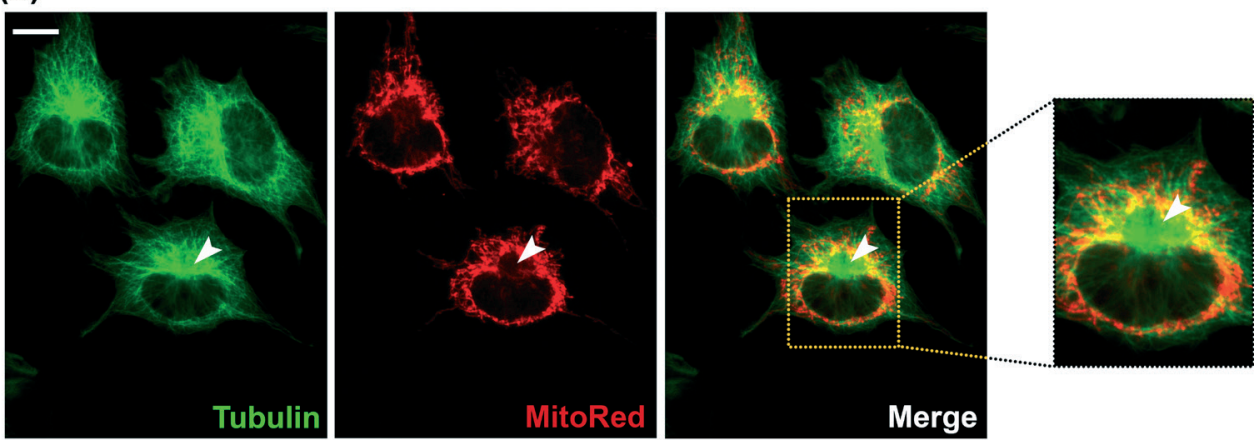

(c)
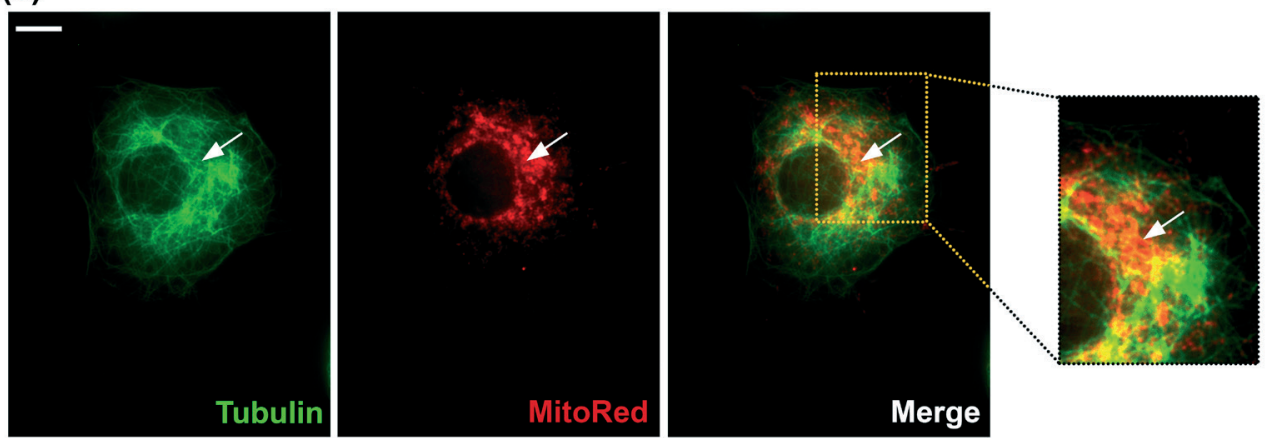

Figure 1. Mitochondrial morphology in control uninfected L929 cells (a, b) and localization of MTOC in control uninfected cells (a, b) and loss of the MTOC at 18 h.p.i. with ECTV-MOS (c).

$(\mathbf{a}, \mathbf{b})$ arrowheads show the MTOC (area without mitochondria), (c) arrows indicate aggregation of mitochondria in place of MTOC. Nuclei (blue), a-tubulin (green), mitochondria (red). Scale bars: $10 \mu \mathrm{m}(\mathbf{a}), 25 \mu \mathrm{m}(\mathbf{b}, \mathbf{c})$. 
(a)
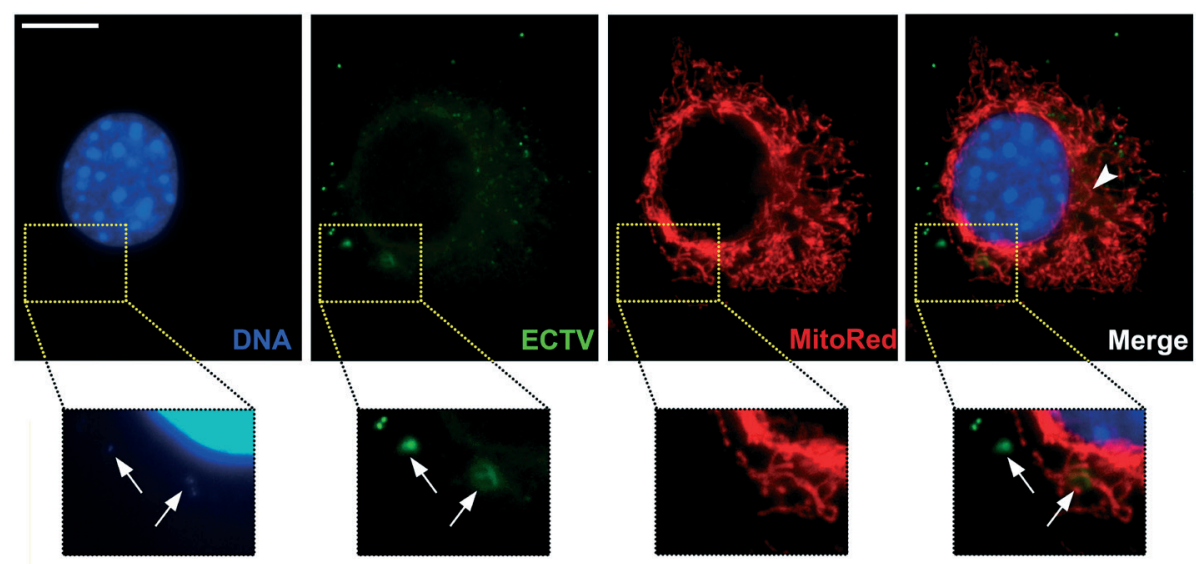

(b)
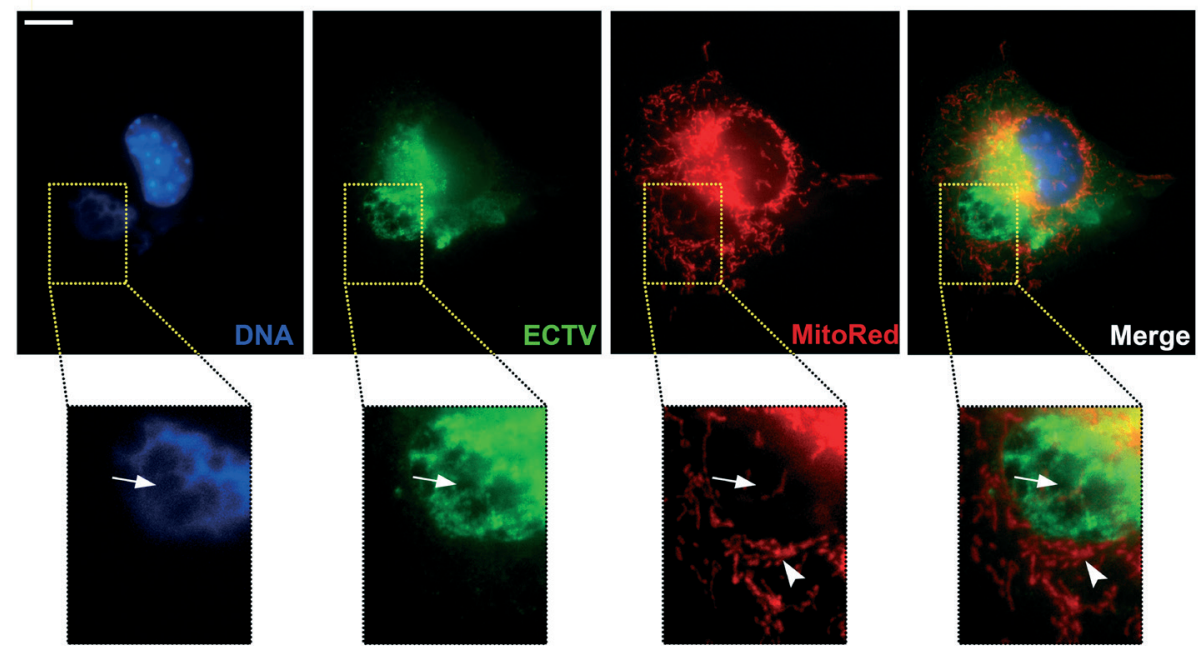

Figure 2. Mitochondrial network organization in infected L929 cells at 4 (a) and 8 (b) h.p.i. with ECTV-MOS.

(a) arrowhead shows MTOC, arrows indicate co-localization of viral DNA with antibodies directed against ECTV-MOS. (b) arrowheads indicate localization of mitochondria around a viral factory, arrows show viral factory formed in the cytoplasm of the infected cell. Nuclei (blue), viral antigens (green), mitochondria (red). Scale bars: $10 \mu \mathrm{m}$.

tion, implying that ECTV-MOS "prepares" mitochondria for participation in viral replication. It is likely that these organelles may supply energy for viral replication and maturation as suggested for ASFV infection (Rojo et al., 1998). In our future research, we plan to check the level of ATP during ECTV-MOS infection in L929 cells which will be able to give us an information about ECTV-MOS-mediated changes in the level of cellular energy. These results will be essential for a better understanding of mitochondrial participation in the replication and maturation of ECTV-MOS. On the other hand, orthopoxviruses are known to inhibit programmed cell death (Everett et al., 2000; Wasilenko et al., 2003). For that reason, ECTV-MOS may contribute to the accumulation of mitochondria thus preventing the release of mediators of apoptosis. Such behaviour of mitochondria has been reported during hepatitis $\mathrm{B}(\mathrm{HBV})$ or $\mathrm{C}(\mathrm{HCV})$ virus infection (Nomura-Takigawa et al., 2006; Kim et al., 2007). Our previous studies (Krzyżowska et al., 2002) show that ECTV-MOS modulates apoptosis of L929 and RK13 cells through inhibition of caspase activity (caspase 3 and 7) before successful virus replication is completed. Within the first few hours of infection (2-8 h.p.i.) there are no significant differences in the percentage of apoptotic cells between infected and uninfected control cells. However, release of the first ECTV-MOS progeny from infected cells may signal to stop inhibition of apoptosis by ECTV-MOS-encoded proteins and consequently increase the percentage of apoptotic cells, as seen between 12 and 18 h.p.i, compared to uninfected control cells (Krzyżowska et al., 2002). Nevertheless, caspase 3 and 7 are executive caspases in apoptosis and are common to both intrinsic (mitochondrial) and extrinsic pathways of this type of cell death. Therefore, in the future studies, we will focus on examining the proteins of the mitochondrial pathway of apoptosis in order to determine the importance of mitochondria in the ECTV-MOS replication cycle.

With regard to the ability to manipulate cells, VACV and ECTV-MOS may be involved in mitochondrial recruitment to the proximity of viral factories and responsible for their fragmentation to inhibit antiviral factors during infection. It is highly likely that ECTV-MOS exploits mitochondrial energy production, and has capability to inhibit antiviral innate immunity. 
(a)
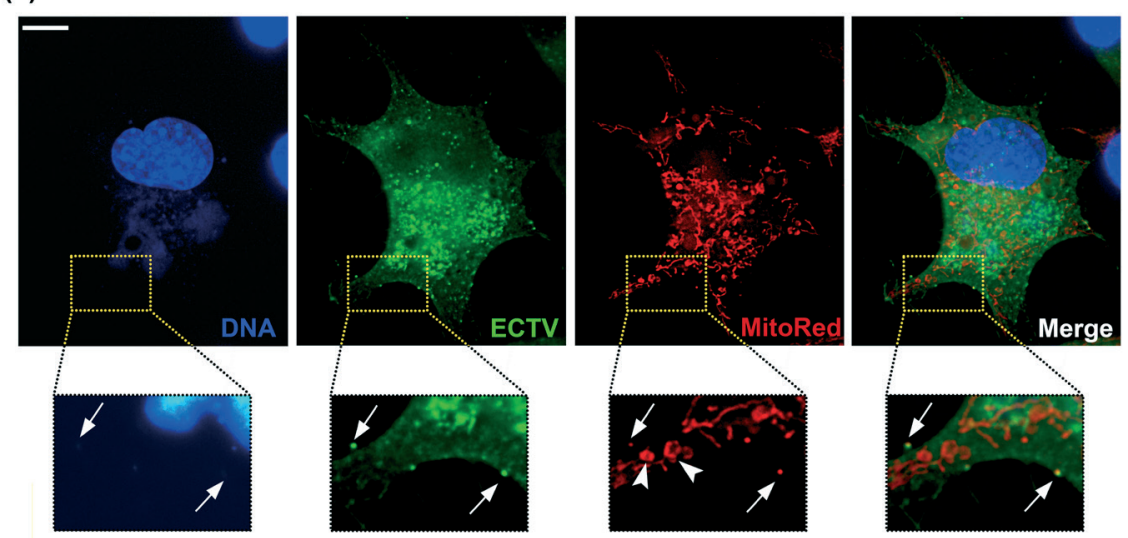

(b)
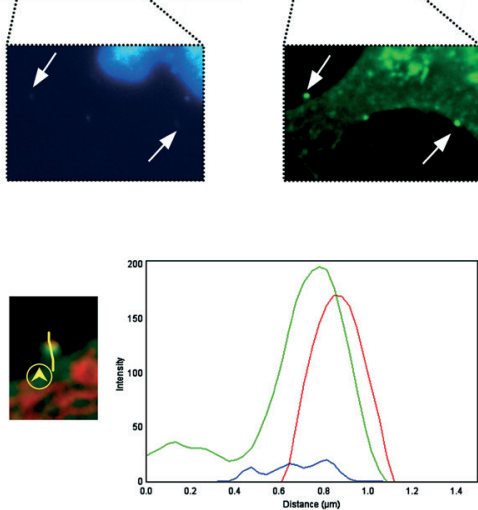

(d)
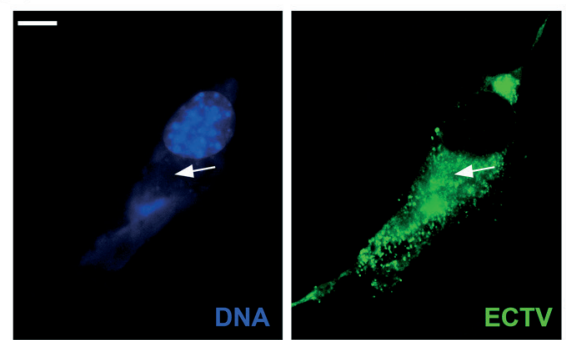
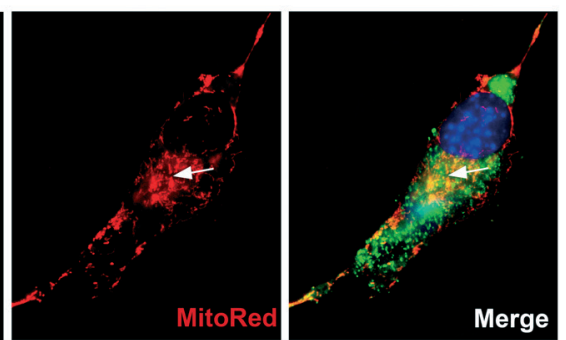

(c)

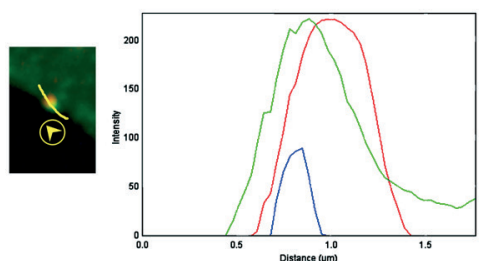

Figure 3. Disruption of mitochondrial network in L929 cells at 18 (a) and 24 (d) h.p.i. with ECTV-MOS.

(a) arrowheads show donut-like mitochondria, arrows indicate co-localization of progeny virions with discrete mitochondria. (b, c) histograms showing relative fluorescence intensity measured along sites of co-localization of a viral particle and mitochondria. (d) arrows show localization of mitochondria between nucleus and viral factory. Nuclei (blue), viral antigens (green), mitochondria (red). Scale bars: $10 \mu \mathrm{m}$.

\section{ECTV-MOS is responsible for destruction of mitochondrial network in permissive L929 cells during the late phase of infection}

It is well known that mitochondria are highly dynamic organelles that frequently divide and fuse. Changes of mitochondrial network depend on physiological or pathological conditions of mitochondrial metabolism. Under normal conditions, mitochondrial fragmentation contributes mitochondria for the daughter cells during cell division and improves distribution of mitochondria along microtubule tracks. However, severe cellular stress leads to mitochondrial fission and consequently to the selective removal of dividing mitochondria by an autophagy process, termed mitophagy. The intensification of mitochondrial division during severe stress may lead to apoptotic cell death (Bossy-Wetzel et al., 2003).

Our findings indicate that ECTV-MOS contributes to destruction of mitochondrial network in L929 cells during late stages of infection. We detected the dispersal of mitochondria at 18 and 24 h.p.i. (Fig. 3a, 3d). Schepis and colleagues (2006) have shown that VACV leads to the collapse of mitochondria also in the early hours after infection. The discrepancy between those results and ours may be due to different rates of ECTV-MOS and VACV replication. At 4 h.p.i. we observed only single ECTV virions on/inside the cell, without viral factories, in contrast to VACV-infected cells (Schepis et al., 2006; Lynn et al., 2012) where viral factories as well as mitochondrial changes were detected at the indicated time. Recent studies by Lynn et al. (2012) have shown that replication of ECTV is approximately 1.5-fold slower than that of VACV. Probably, this accounted for the observation of intact mitochondria at early time points following infection with ECTV.

In our studies, at subsequent time points, i.e. 18 and 24 h.p.i., mitochondria displayed 4 types of morphological forms in ECTV-MOS-infected L929 cells: 1) loose mitochondrial network ("loose net"), when interconnected network is relaxed, 2) short, individual mitochondrial tubules, 3) donut-like mitochondria, and 4) single, separated, circular mitochondria (discrete mitochondria) (Fig. 3a). Each form is characteristic for particular cellular stress. Mild oxidative stress may lead to formation of donut-like mitochondria, but high oxidative stress extensively destroys the mitochondrial network leaving only discrete mitochondria (Ahmad et al., 2013). Additionally, donut-like mitochondria could result from hypoxia-re- 
(a)

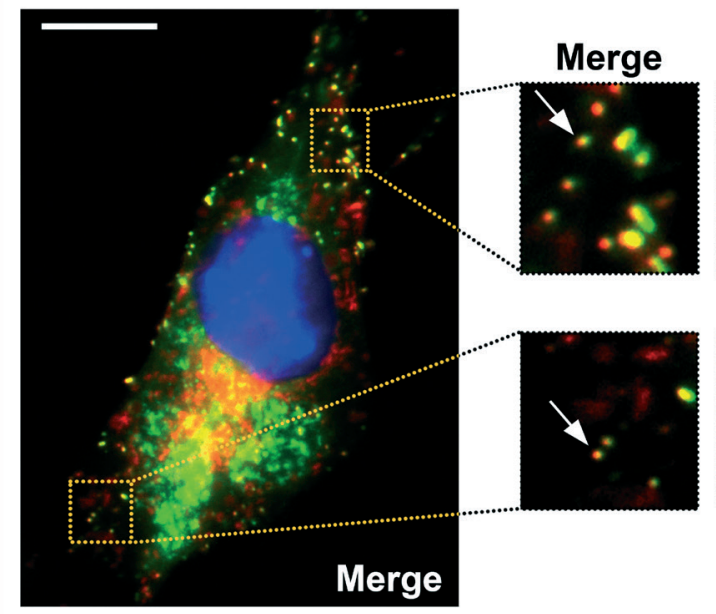

(b)

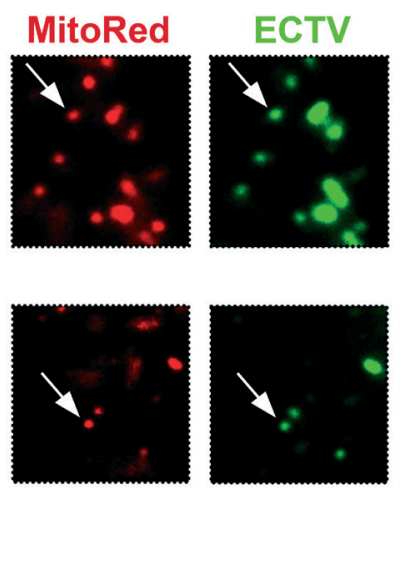

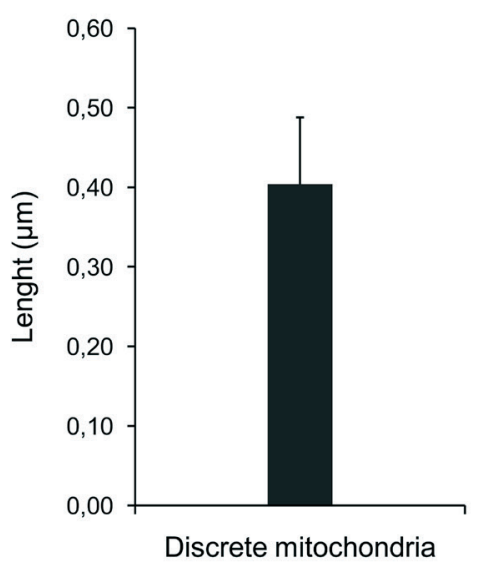

Figure 4. Co-localization of discrete mitochondria and progeny virions in L929 cells at 18 h.p.i. with ECTV-MOS.

(a) arrows indicate co-localization of progeny virions with discrete mitochondria. (b) bar shows length ( $\mu$ m) of discrete mitochondria colocalized with virions (150 samples). Nuclei (blue), viral antigens (green), mitochondria (red). Scale bars: $10 \mu \mathrm{m}$.

oxygenation stress, when anomalous fusion events are favored (fusion of 2-3 mitochondria) (Liu \& Hajnóczky, 2011). Available evidence shows that fragmented mitochondria lose their membrane potential, however, donutlike mitochondria quickly regain the membrane potential and so retain competence, thus preserving the organelles under conditions of metabolic stress. This may explain the donut-like mitochondria formation during ECTVMOS infection where apoptosis is not instantly evident and it is known that the loss of mitochondrial potential can led to apoptosis. Moreover, recent research has revealed that VACV creates hypoxic response under normal oxygen levels that imitates the situation in solid tumors (Mazzon et al., 2013). Cells predominantly produce energy by a high rate of glycolysis and reduce oxidative phosphorylation during hypoxia. This observation, described as a Warburg effect, may simply be a consequence of mitochondrial metabolism reduction, which limits reactive oxygen species (ROS) production, and then protects the cell from death (Mazzon et al., 2013). Interestingly, donut-like mitochondria, which form during hypoxia, and discrete mitochondria are the main source of ROS. As both donut-like mitochondria and small, fragmented mitochondria are observed during late stages of ECTV-MOS infection, we hypothesize that ROS may accumulate in a cell after the replication and assembly of progeny virions, but this requires further investigation. On the other hand, the mitochondrial network disintegrates into shorter fragments although not completely. The number of donut-like or discrete mitochondria is quite inconsiderable compared to short tubules and loose net in ECTV-MOS-infected L929 cells at 18 and 24 h.p.i., which may suggest that the virus prevents full mitochondrial degradation and protects the cell from ROS accumulation and finally from death.

\section{Progeny virions of ECTV-MOS co-localize with discrete mitochondria in $\mathbf{L} 929$ cells during late stages of infection}

Mitochondrial transport depends on both actin and tubulin cytoskeleton in mammalian cells (Morris \& Hollenbeck, 1995; Ligon \& Steward, 2000; Hollenbeck \&
Saxton, 2005). Physiological shift between mitochondrial network and mitochondrial particles closely relates to the need for organelle trafficking. When mitochondrial fission is blocked, the network appears to be branched and mitochondrial movement along actin cables is abolished (Varadi et al., 2004). These findings demonstrate that only fragmented mitochondria are able to be transported throughout the cell.

Destruction of mitochondrial network in ECTVMOS-infected L929 cells leads to the formation of small discrete mitochondria (Figs. 3a-c, 4). Interestingly, these structures were observed only during the late stage of ECTV-MOS infection, when progeny viral particles localize near the cellular membrane. Our observations have led to a surprising discovery that discrete mitochondria localize in close association with ECTV-MOS virions (Figs. 3a-c, 4a). Discrete mitochondria are approximately $400 \mathrm{~nm}$ in length (Fig. 4b). This is consistent with our previous study, which indicated that the actin tails or filopodia with viral particle/s are formed during ECTV-MOS infection in permissive Vero and BALB/3T3 cells (Boratyńska et al., 2010). The actin tails and filopodia consist of actin filaments and are important in the spread of ECTV from cell to cell in vitro. Besides their participation in mitochondrial transport under normal conditions, a role for actin filaments is implied in viral infections. This suggests that discrete mitochondria may be important for the transportation of mature progeny virions using actin filaments.

In conclusion, our study shows that ECTV-MOS contributes to changes in the mitochondrial network in permissive L929 cells during infection. These changes are manifested by destruction of the mitochondrial network as well as loosening of the connections between individual mitochondrial tubules. Mitochondrial intracellular distribution is also rearranged upon infection with ECTVMOS. These findings suggest a strategy for orthopoxviruses to effectively escape from mitochondria-mediated innate immunity. Furthermore, our findings point to the possibility that ECTV-MOS may use mitochondria for energy requirements of replication or viral particle transportation using the cytoskeleton. 


\section{Acknowledgements}

We thank Felix N. Toka (Ph.D. from the WULSSGGW) for critical reading of the manuscript and valuable remarks.

This research was supported by the grant No. 2011/03/B/NZ6/03856 from the National Science Center in Cracow, Poland (to $\mathrm{MN}$ ).

\section{REFERENCES}

Ahmad T, Aggarwal K, Pattnaik B, Mukherjee S, Sethi T, Tiwari BK, Kumar M, Micheal A, Mabalirajan U, Ghosh B, Sinha Roy S, Agrawal A (2013) Computational classification of mitochondrial shapes reflects stress and redox state. Cell Death Dis 4: e461.

Arnoult D (2007) Mitochondrial fragmentation in apoptosis. Trends Cell Biol 17: 6-12.

Benard G, Bellance N, James D, Parrone P, Fernandez H, Letellier T, Rossignol R (2007) Mitochondrial bioenergetics and structural network organization. J Cell Sci 120: 838-848.

Bereiter-Hahn J, Voth M (1994) Dynamics ofmitochondria in living cells: shape changes, dislocations, fusion, and fission of mitochondria. Microsc Res Tech 27: 198-219.

Boldogh IR, Pon LA (2006) Interactions of mitochondria with the actin cytoskeleton. Biochim Biophys Acta 1763: 450-462.

Boratyńska A, Martyniszyn L, Szulc L, Krzyżowska M, Szczepanowska J, Niemiałtowski MG (2010) Contribution of rearranged actin structures to the spread of Ectromelia virus infection in vitro. Acta Virol 54: 41-48.

Bossy-Wetzel E, Barsoum MJ, Godzik A, Schwarzenbacher R, Lipton SA (2003) Mitochondrial fission in apoptosis, neurodegeneration and aging. CurrOpin Cell Biol 15: 706-716.

Chen RA, Ryzhakov G, Cooray S, Randow F, Smith GL (2008) Inhibition of IkappaB kinase by vaccinia virus virulence factor B14. PLoS Pathog 4: e22.

Deng L, Dai P, Parikh T, Cao H, Bhoj V, Sun Q, Chen Z, Merghoub T, Houghton A, Shuman S (2008) Vaccinia virus subverts a mitochondrial antiviral signaling protein-dependent innate immune response in keratinocytes through its double-stranded RNA binding protein, E3. I Virol 82: 10735-10746.

Everett H, Barry M, Lee SF, Sun XJ, Graham K, Stone I, Bleackley RC, McFadden G (2000) M11L: A novel mitochondria-localized protein of myxoma virus that blocks apoptosis in infected leukocytes. J Exp Med 191: 1487-1498.

Fenner F (2000) Adventures with poxviruses of vertebrates. FEMS Microbiol Rev 24: 123-133.

Gomes LC, Di Benedetto G, Scorrano L (2011) During autophagy mitochondria elongate, are spared from degradation and sustain cell viability. Nat Cell Biol 13: 589-598.

Guzun R, Karu-Varikmaa M, Gonzalez-Granillo M, Kuznetsov AV, Michel L, Cottet-Rousselle C, Saaremäe M, Kaambre T, Metsis M, Grimm M, Auffray C, Saks V (2011) Mitochondria-cytoskeleton interaction: distribution of $\beta$-tubulins in cardiomyocytes and HL-1 cells. BiochimBiophys Acta 1807: 458-469.

Heath CM, Windsor M, Wileman T (2001) Aggresomes resemble sites specialized for virus assembly. J Cell Biol 153: 449-455.

Hollenbeck PJ \& Saxton WM (2005) The axonal transport of mitochondria. J Cell Sci 118: 5411-5419.

Ishikawa H, Barber GN (2008) STING is an endoplasmic reticulum adaptor that facilitates innate immune signalling. Nature 455: 674 678 .
Kim S, Kim HY, Lee S, Kim SW, Sohn S, Kim K, Cho H (2007) Hepatitis $B$ virus $x$ protein induces perinuclear mitochondrial clustering in microtubule- and dynein-dependent manners. J Virol 81: 1714-1726.

Krzyżowska M, Schollenberger A, Skierski J, Niemiałtowski M (2002) Apoptosis during ectromeliaorthopoxvirus infection is DEVDase dependent: in vitro and in vivo studies. Microbes Infect 4: 599-611.

Ligon LA \& Steward O (2000) Role of microtubules and actin filaments in the movement of mitochondria in the axons and dendrites of cultured hippocampal neurons. J Comp Neurol 427: 351-361.

Liu X, Hajnóczky G (2011) Altered fusion dynamics underlie unique morphological changes in mitochondria during hypoxia-reoxygenation stress. Cell Death Differ 18: 1561-1572.

Lynn H, Horsington J, Ter LK, Han S, Chew YL, Diefenbach RJ, Way M, Chaudhri G, Karupiah G, Newsome TP (2012) Loss of cytoskeletal transport during egress critically attenuates ectromelia virus infection in vivo. J Virol 86: 7427-7443.

Mazzon M, Peters NE, Loenarz C, Krysztofinska EM, Ember SW, Ferguson BJ, Smith GL (2013) A mechanism for induction of a hypoxic response by vaccinia virus. Proc Natl Acad Sci USA 110: 12444-12449.

McFadden G (2005) Poxvirus tropism. Nat Rev 3: 201-213.

Melo-Silva CR, Tscharke DC, Lobigs M, Koskinen A, Wong YC, Buller RM, Müllbacher A, Regner M (2011) The ectromelia virus SPI-2 protein causes lethal mousepox by preventing NK cell responses. $J$ Virol 85: 11170-11182.

Morris RL \& Hollenbeck PJ (1995) Axonal transport of mitochondria along microtubules and Factin in living vertebrate neurons. J Cell Biol 131: 1315-1326.

Nomura-Takigawa Y, Nagano-Fujii M, Deng L, Kitazawa S, Ishido S, Sada K, Hotta H (2006) Non-structural protein 4A of hepatitis C virus accumulates on mitochondria and renders the cells prone to undergoing mitochondria-mediated apoptosis. J Gen Virol 87: 19351945.

Risco C, Rodríguez JR, López-Iglesias C, Carrascosa JL, Esteban M, Rodríguez D (2002) Endoplasmic reticulum-Golgi intermediate compartment membranes and vimentin filaments participate in vaccinia virus assembly. J Virol 76: 1839-1855.

Rojo G, Chamorro M, Salas ML, Vinuela E, Cuezva JM, Salas J (1998) Migration of mitochondria to viral assembly sites in african swine fever virus-infected cells. J Virol 72: 7583-7588.

Schepis A, Schramm B, de Haan CA, Locker JK (2006) Vaccinia virusinduced microtubule dependent cellular rearrangements. Traffic 7: 308-323.

Seth RB, Sun L, Chen ZJ (2006) Antiviral innate immunity pathways. Cell Res 16: 141-147.

Tait SW, Green DR (2010) Mitochondria and cell death: outer membrane permeabilization and beyond. Nat Rev Mol Cell Biol 11: 621632

Tolonen N, Doglio L, Schleich S, Krijnse Locker J (2001) Vaccinia virus DNA replication occurs in endoplasmic reticulum-enclosed cytoplasmic mini-nuclei. Mol Biol Cell 12: 2031-2046.

Wasilenko ST, Stewart TL, Mevers AF, Barry M (2003) Vaccinia virus encodes a previously uncharacterized mitochondrial-associated inhibitor of apoptosis. Proc Natl Acad Sci 100: 14345-14350.

Wasilewski M, Scorrano L (2009) The changing shape of mitochondrial apoptosis. Trendsv Endocrinol Metab 20: 287-294.

Varadi A, Johnson-Cadwell LI, Cirulli V, Yoon Y, Allan VJ, Rutter GA (2004) Cytoplasmic dynein regulates the subcellular distribution of mitochondria by controlling the recruitment of the fission factor dynamin-related protein-1. J Cell Sci 117: 4389-4400. 